REPRINTED FROM ANNALS OF OTOLOGY, RHINOLOGY \& LARYNGOLOGY, JANUARY-FEBRUARY 1987

Volume 96, Number 1, Part 2

Supplement 128

COPYRIGHT 1987, ANNALS PUBLISHING COMPANY

\title{
MIDDLE EAR INFECTION POSTIMPLANTATION: RESPONSE OF THE ROUND WINDOW MEMBRANE TO STREPTOCOCCUS PYOGENES
}

\author{
N. E. Cranswick, B Med SC; B. K-H. Franz, MD, Dip Aud; G. M. Clark, PhD, FraCs; \\ R. K. SHEPHERD, BSC, DIP ED; D. M. BLOOM, B APP SC
}

Funded by the Deafness Foundation of Victoria, the Channel 10 Deafness Appeal, and the National Health and Medical Research Council of Australia.

The seal of the implanted round window membrane to resist Streptococcus pyogenes invasion from the middle ear was investigated in 12 cats. Results showed that the implanted round window membrane is able to form a barrier for $S$ pyogenes starting 1 week postimplantation. Under normal conditions $S$ pyogenes did not pass through the round window membrane, nor through the gap that existed between the membrane and the prosthesis. Mechanical disruption of the round window seal, however, and severe inflammatory response to $S$ pyogenes caused the infection to extend into the inner ear.

One of the problems associated with intracochlear prostheses is that of middle ear infection postimplantation. An adequate round window seal is regarded as essential to form a barrier for microorganisms or toxins that could enter the inner ear from an infected middle ear. It has been estimated that over $90 \%$ of children have at least one episode of otitis media in the first 6 years of life. ${ }^{1}$ It is in this group in particular that cochlear implants could be most beneficial. Widely based experimental data therefore must be obtained before considering young children for cochlear implantation.

The effects of Streptococcus pyogenes (unpublished data), Staphylococcus aureus, ${ }^{2}$ and recently Pseudomonas aeruginosa and Streptococcus pneumoniae $e^{3.4}$ have been investigated in implanted cat cochleas. Streptococcus pyogenes and $S$ aureus failed to show adverse effects in implanted cochleas, although an increase of the round window permeability was observed. ${ }^{2.5}$ Pseudomonas aeruginosa and $S$ pneumoniae demonstrated pathological changes in the implanted, but also in the nonimplanted cochlea. A clear conclusion, however, as to whether the implanted cochlea was at a disadvantage could not be drawn because of the methodical limitations of these studies.

To understand more about the sealing mechanisms of the implanted round window membrane a further study was carried out using $S$ pyogenes to induce a purulent otitis media in the implanted cochlea.

\section{METHODS}

Twelve healthy adult cats were implanted bilaterally with dummy scala tympani electrodes. Eight animals were induced with streptococcal (S pyogenes) middle ear infections at different intervals postimplantation (l week, 2 weeks, 4 weeks, 8 weeks) by transbulla inoculation. Four cats were implanted and killed at times corresponding to those of the inoculated animals, and were used as a control group. Thus a comparison between the histology of infected and uninfected ears postimplantation could be made. In each animal one implanted electrode consisted of plain Silastic, whereas the electrode implanted within the other cochlea contained multiple platinum rings. Inoculation was carried out with a suspension of $10^{8}$ organisms $/ \mathrm{mL}$ in a nutrient broth. This was introduced directly into the surgically opened bulla, which was packed with Gelfoam to retain the bacteria. About $0.5 \mathrm{~mL}$ was inserted into each bulla. The animals were killed by intra-arterial perfusion 1 week after inoculation. Cochlear specimens were fixed in Karnovsky's fixative, decalcified in EDTA, and embedded in Spurr's resin. Thin sections ( $2 \mu \mathrm{m}$ thick) were cut every $30 \mu \mathrm{m}$ until a midmodiolar section was obtained. Sections were stained with hematoxylin and eosin, and representative sections stained by Gram's technique to identify organisms. Specimens from surgical procedures were routinely plated out on horse blood agar and MacConkey's medium. Initially specimens were stored in Stuart's transport medium before plating out. Later specimens were plated out immediately at killing. Organisms were identified on the basis of growth characteristics on the two different media, the size and shape of the colonies, and the Gram's stain properties of the bacteria. Additionally, serotyping was carried out using the Streptex test.

\section{RESULTS}

In five implanted cochleas an effective barrier for $S p y$ ogenes was formed between the middle and inner ear by the metaplastic round window membrane and the adjacent electrode. Two types of seals were observed. One consisted of metaplastic epithelium that extended across the round window membrane and escorted the electrode a short distance into the cochlea before merging with a pseudostratified squamous epithelium and the endothelial lining of the scala tympani. The other, occasionally seen, showed a fibrous tissue envelope around the implant. This envelope was lined by eosinophilic squamous cells that were often in continuum with the metaplastic epithelium of the round window membrane. The result was essentially externalization of the electrode into the middle ear space.

Three inoculated cochleas, however, showed evidence of severe infection that had spread through the round window membrane into the inner ear, thus causing labyrinthitis. This form of complication was seen in the longer term animals when inoculation was carried out 4 or 8 weeks after implantation, and was thought to be caused by disruption of the round window seal when the electrode was disturbed during the inoculation procedure. In the shorter term animals, ie, 1 or 2 weeks after implantation, a period 
regarded as more vulnerable to infection, invasion of bacteria was limited to the area around the round window membrane and infection was not present within the inner ears.

The cochleas implanted with platinum rings showed a slightly increased inflammatory response in the region of the rings. There was, however, no observable difference between the resistance of the two electrode designs.

Characteristically the infected round window membrane showed metaplasia of the epithelial layer to a tall columnar-type respiratory epithelium with many ciliated and secretory cells. The middle layer of the round window had become thickened with edema, an inflammatory cell infiltrate, and an increase in fibrous tissue elements within the deeper portion of the membrane. New bone formation was observed in cochleas that were implanted for 8 weeks. Osteoneogenesis was not only seen within the cochlea, but also at the round window membrane.

Loss of hair cells in the inoculated implanted cochleas were either related to trauma or infection within the cochlea. When the loss was the result of trauma, it was usually localized to the site of trauma. However, infection within the cochlea resulted in the loss of hair cells throughout the cochlea. Parallel findings were observed with regard to the loss of neural fibers and ganglion cells.

Initial cultures obtained from inoculated cochleas (five cats) did not reveal $S$ pyogenes when stored in Stuart's transport medium before plating out on horse blood agar and MacConkey's medium. However, subsequent cultures (three cats) showed growth of $S$ pyogenes when plated out on these media immediately at killing. Microscopically, gram-positive organisms were seen to be restricted to the epithelial layer of the round window membrane. In those cases of labyrinthitis, microorganisms were also seen within the cochlea.

\section{DISCUSSION}

One of the questions in cochlear implantation that needs to be answered is whether the round window seal can resist bacterial invasion into the cochlea. This is particularly important when children are considered for implantation, because they have a higher incidence of otitis media than adults, but also form a group that would benefit the most from implantation.

Critical areas for bacterial invasion into the inner ear after implantation are seen in the round window membrane itself, with its increased permeability, and in the gap that exists between the round window membrane and the prosthesis. Increased permeability is an initial response of the implanted round window membrane observed over a period of 2 weeks postimplantation. ${ }^{5}$ Increased vulnerability of the inner ear can be expected over this period. The gap between the window membrane and the prosthesis, however, appears to persist, and remains a potential entrance for bacteria and toxins (unpublished data).

Despite increased permeability and a persisting gap between the membrane and the prosthesis of implanted cochleas, streptococcal infection did not cause any further hair cell and nerve fiber losses. Microorganisms were not seen beyond the epithelial layer of the round window membrane, nor within the gap it formed with the prosthesis. It seems therefore that the implanted round window membrane can form a barrier for bacterial and toxic invasion, and that other additional mechanisms are present that might explain this defense mechanism.

In this study we found basically two types of seals in the implanted round window membrane. In both, metaplastic epithelium consisting of ciliated and goblet cells had moved across the round window membrane along the electrode for a short distance into the cochlea before merging with an eosinophilic pseudostratified epithelium. The latter merged with the endothelial lining of the scala tympani in one seal, and in the other with the fibrous tissue envelope that surrounded the implant.

The absence of microorganisms in the gap between the membrane and the prosthesis suggests that the cilia were able to repel invading organisms while at the same time a mucoid layer produced by the goblet cells prevented the bacteria from contacting the epithelial cells. Thus $S p y$ ogenes, having a number of enzymes to support the spread of microorganisms in tissue, was practically unsuccessful in entering the inner ear.

In three implanted cochleas, however, a bacterial labyrinthitis was observed. All three cochleas showed a severe inflammatory reaction in the middle ear that had spread around the prosthesis into the inner ear with consequent hair cell and nerve fiber losses. Gram-positive microorganisms were not only seen in the middle ear cleft but also within the labyrinth. Notably these cochleas belonged to the longer term animals that were inoculated 4 and 8 weeks after implantation, a period regarded as more than sufficient for the round window membrane to heal.

An explanation for the labyrinthitis is seen in a possible mechanical disruption of the already formed round window seal at the time of inoculation. Another explanation could be the extraordinary severity of the inflammation in the middle ear cleft and the reduced resistance of these animals to streptococcal infection.

\section{CONCLUSIONS}

The implanted round window membrane of the cat forms a barrier for $S$ pyogenes infection starting 1 week after implantation. Respiratory epithelium extends across the round window membrane and escorts the electrode into the scala tympani. Severe inflammatory reactions to infection in the middle ear or mechanical disruption of an already established seal in the implanted round window membrane may promote the spread of infection into the inner ear.

ACKNOWLEDGMENT - We thank Dr Robbins-Browne, Department of Microbiology, University of Melbourne. for the supply of the bacteria used in this study.

\section{REFERENCES}

1. Giebink GS, Ouie PG. Otitis media: the spectrum of middle ear inflammation. Ann Rev Med 1978;29:285-306.

2. Clark GM, Shepherd RK. Cochlear implant and round window sealing procedures in the cat. Acta Otolaryngol [Suppl] (Stockh) 1984(suppl 410):5-15.

3. Berkowitz RG, Franz BK-H, Shepberd RK, Clark GM, Bloom DM. Cochlear implant and otitis media: a pilot study to assess the feasibility of pseudomonas aeruginosa and streptococcus pneumoniae infection in the cat. J Otolaryngol Soc Aust 1985:5:297-9.

4. Berkowitz RG, Franz BK-H, Shepherd RK, Clark GM, Bloom DM. Pneumococcal middle ear infection and cochlear implantation. Ann Otol Rhinol Laryngol 1987;96:(suppl 128):55-6.

5. Franz BK-H, Clark GM. Bloom DM. Permeability of the implanted round window membrane in the cat. Acta Otolaryngol [Suppl] (Stockh) 1984(suppl 410):17-23. 


\section{University Library}

\section{- M M I E E R VA A gateway to Melbourne's research publications}

Minerva Access is the Institutional Repository of The University of Melbourne

Author/s:

Cranswick, N. E.;Franz, B. K-H.;Clark, Graeme M.;Shepherd, R. K.;Bloom, D. M.

Title:

Middle ear infection postimplantation: response of the round window membrane to Streptococcus pyogenes

Date:

1987

Citation:

Cranswick, N. E., Franz, B. K., Clark, G. M., Shepherd, R. K. \& Bloom, D. M. (1987). Middle ear infection postimplantation: response of the round window membrane to Streptococcus pyogenes. Annals of Otology, Rhinology \& Laryngology, January-February, 96(1, part 2, suppl.128), 53-54.

Persistent Link:

http://hdl.handle.net/11343/27245 